Check for updates

Cite this: RSC Adv., 2019, 9, 27264

\title{
Model Affitin and PEG modifications onto siRNA lipid nanocapsules: cell uptake and in vivo biodistribution improvements
}

\author{
Pauline Resnier, ${ }^{a}$ Elise Lepeltier, (iD ${ }^{a}$ Anthea Lucrezia Emina, ${ }^{a}$ Natacha Galopin, ${ }^{b}$ \\ Jérôme Bejaud, ${ }^{a}$ Stephanie David, ${ }^{c}$ Caroline Ballet, ${ }^{d}$ Thierry Benvegnu, ${ }^{d}$

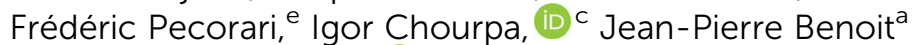 \\ and Catherine Passirani (D) *a
}

Malignant melanoma is an aggressive tumor, associated with the presence of local and/or distant metastases. The development of gene therapy by the use of small interfering RNA (siRNA) represents a promising new treatment. However, the protection of this biomolecule is necessary in order for it to be intravenously administrated, for example via its incorporation into nanomedicines. In parallel to the passive targeting usually obtained by pegylation, various studies have aimed at developing "smart" nanomedicines to efficiently deliver the drug to tumor sites. In this work, siRNA loaded lipid nanocapsules (LNCS) were modified with DSPE-polyethylene glycol (DSPE-PEG), tetraether-PEG (TE-PEG) and/or with an Affitin model, to assay multiple targeting strategies. The uptake of fluorescently labelled LNCs, nanocarrier integrity and siRNA release into human SKMel28 melanoma cells were studied by flow cytometry, conventional confocal microscopy and by confocal spectral imaging in a Förster Resonance Energy Transfer (FRET) mode. Surface modified siRNA LNCs were followed after human plasma incubation and after intravenous injection, in order to compare the stealth properties. Finally, the biodistribution of the different siRNA LNCs in healthy and melanoma tumor bearing mice models was assessed by in vivo biofluorescence imaging (BFI), to evaluate the potential tumor targeting ability. The post-insertion of DSPE-PEG induced a strong decrease of the internalization into melanoma cells compared to TE-PEG modification. Both PEG polymer decorations induced a great plasma protection of siRNA but only DSPE-PEG led to stealth properties, even at low concentration ( $5 \mathrm{mM}$ ). The Affitin grafting by thiolation of DSPE-PEG was validated on siRNA LNCS. DSPE-PEG-Affitin LNCs were not detected in this melanoma tumor model but did not show unspecific accumulation in organs. DSPE-PEG and TE-PEG LNCS induced a significant intratumoral accumulation of modified LNCS.

Received 15th May 2019

Accepted 6th August 2019

DOI: $10.1039 / \mathrm{c} 9 \mathrm{ra03668g}$

rsc.li/rsc-advances by RISC (RNA-induced silencing complex) pathways leading to transitory inhibition of protein production in the cytoplasm. ${ }^{1}$ However, this hydrophilic entity is not able to cross the cellular membrane alone and it is fragile.

For a few decades now, the encapsulation or adsorption of siRNA have been mainly based on electrostatic interactions of anionic nucleic acids with cationic lipids or polymers such as DOTAP (1,2-dioleoyl-3-trimethylammoniumpropane), cholesterol, or polyethylenimine (PEI), respectively forming lipoplexes and polyplexes. ${ }^{2,3}$ Neutral or zwitterionic lipids, such as DOPE (1,2-dioleoyl-sn-glycero-3-phosphoethanolamine), are usually added for their fusiogenic property, promoting the endosomal escape into the intracellular compartment. ${ }^{4,5}$ Various nanomedicines, combining these different lipids and/or polymers, have been developed and they have demonstrated protection effects against enzymatic degradation, siRNA crossing through cell membrane and its endosomal escape.$^{6-8}$ Among them, lipid nanocapsules (LNCs), based on emulsion phase inversion process, were adapted for siRNA encapsulation thanks to the 
use of DOTAP/DOPE lipids. ${ }^{\text {9,10 }}$ This LNC formulation is solventfree, low-energy consuming, easily realizable and reproducible inducing stable siRNA complexation and monodispersed formulation. Moreover, LNCs showed an efficient transfection efficacy of siRNA on U87MG glioma and SK-Mel28 melanoma cells with a significant inhibition of two different targeted proteins. ${ }^{9,11}$ With this proof of in vitro transfection, the challenge will be to preferentially accumulate the LNCs into the targeted tumor site, for an efficient in vivo siRNA delivery and a potential therapeutic application.

In the context of melanoma metastases, the intravenous injection route appears as an evidence. In this case siRNA encapsulation into LNCs should provide: (i) protection against blood nucleases and immune cells, (ii) accumulation into tumor site and (iii) penetration into the intracellular compartment.

The formulation of nanoparticles usually includes pharmacokinetic modulating moieties such as polyethylene glycol (PEG). In this study, the surface of the LNCs was modified by different molecules. The pegylation, i.e. the addition of long chains of polyethylene glycol (PEG), is known to play an important role in the efficacy of passive targeting strategy in solid tumor models. ${ }^{12,13}$ This hydrophilic polymer allows a long blood circulation time in limiting the LNC recognition and the massive destruction by the innate immune system and then the elimination via the liver. LNCs already showed their capacity to accumulate into tumor microenvironment, characterized by leaky junctions of the neovessels and a poor lymphatic drainage, a phenomenon known as the EPR effect (enhanced permeability and retention effect). ${ }^{14}$ DSPE-PEG (or 1,2-distearoyl-sn-glycero-3phosphoethanolamine- $N$-[methoxy(polyethyleneglycol)-2000]) has been usually used in LNCs to improve this passive targeting.

In parallel, emerging archaeal tetraether PEG (TE-PEG) molecules are able to form stable and stealth liposomes. ${ }^{\mathbf{1 5 , 1 6}}$ The TE natural lipid has shown a strong stability in several conditions such as high temperature, acidic $\mathrm{pH}$ and serum media. ${ }^{17}$ This lipid found in archaea has been studied for a potential application in drug/gene delivery system. It could be a promising candidate for a new LNC surface modification.

In order to overcome the limits of passive targeting, the active targeting strategies by post-insertion of innovative moieties into nanoparticles to improve their half-life time, their targeting efficiency or stimuli-sensitivity, and their intracellular delivery is a hot topic. ${ }^{18}$ For instance, ligands as sugars, peptides or antibodies grafted on nanomedicine surface are used to specifically recognize tumor antigens. Archaeal extremophilic proteins from the Sul7d family, ${ }^{19}$ such as Sac7d, were recently developed as scaffolding to generate small artificial affinity proteins (Affitins).$^{20-28}$ Affitins appear as an alternative to the use of antibodies for an active targeting approach.

In this study, surface modifications of siRNA LNCs were performed with an innovative TE-PEG polymer and an Affitin model, compared with a classic DSPE-PEG LNCs, in order to evaluate the potential tumor targeting of siRNA LNCs, after intravenous administration: Fig. 1. Flow cytometry and confocal fluorescence microscopy were used to determine the LNC internalization capacity in SK-Mel28 human melanoma cells. The LNC and siRNA integrities after SK-Mel28 incubation was evaluated by fluorescence confocal spectral imaging (FCSI) using the co-loading of two fluorophores: a FRET couple (DiI/DiD) and a fluorescently labelled SiRNA (siRNA Alexa488) were used. Moreover, the protection against nucleases and blood behavior were determined in vitro and in vivo on mice. Finally, biodistribution studies of modified siRNA LNCs on healthy and melanoma tumor grafted nude mice were performed to evaluate the elimination and the potential tumor targeting of these surface modified LNCs.

\section{Results}

\section{Formulation and stability of siRNA LNCs}

The encapsulation of siRNA into LNCs was performed with or without lecithin (Lipoid $\AA)$. The size and zeta potential stability were followed over time after formulation and storage at $4{ }^{\circ} \mathrm{C}$ (Fig. 2). The absence of lecithin into the siRNA loaded LNCs induced a long term stability compared to the initial formulation containing Lipoid ${ }^{\circledR}$. Indeed, the formulation with lipoid $®$, adapted from the initial LNC process, led to an important size decrease and a zeta potential increase: at day 0 , the diameter was around $100 \mathrm{~nm}$ and reached $60 \mathrm{~nm}$ after 15 days, with a zeta potential near neutrality at day 0 and a zeta potential around $+40 \mathrm{mV}$ after 2 weeks. The optimization of the process, by Lipoid ${ }^{\circledR}$ elimination, led to a long-term stability of size and zeta potential, for at least one month with a mean diameter of $80 \mathrm{~nm}$ and a zeta potential of around $+23 \mathrm{mV}$. That is why, in this study, siRNA LNCs were formulated without Lipoid $®$ for all the following experiments.

\section{Behavior of FRET siRNA LNCs in melanoma cells}

siRNA delivery by LNCs was studied on living melanoma cells by fluorescence confocal multispectral imaging: FCSI (Fig. 3). In

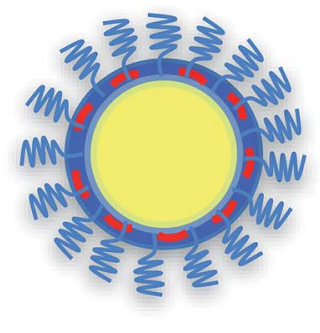

DSPE-PEG LNC

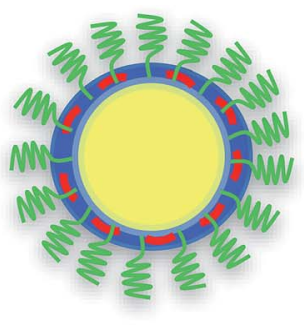

TE-PEG LNC

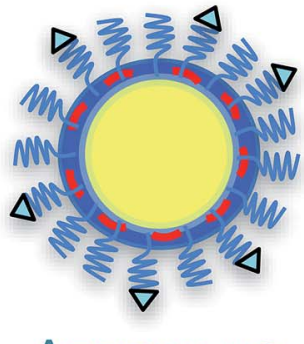

AFFITIN LNC

Fig. 1 The different performed and studied surface functionalizations. 

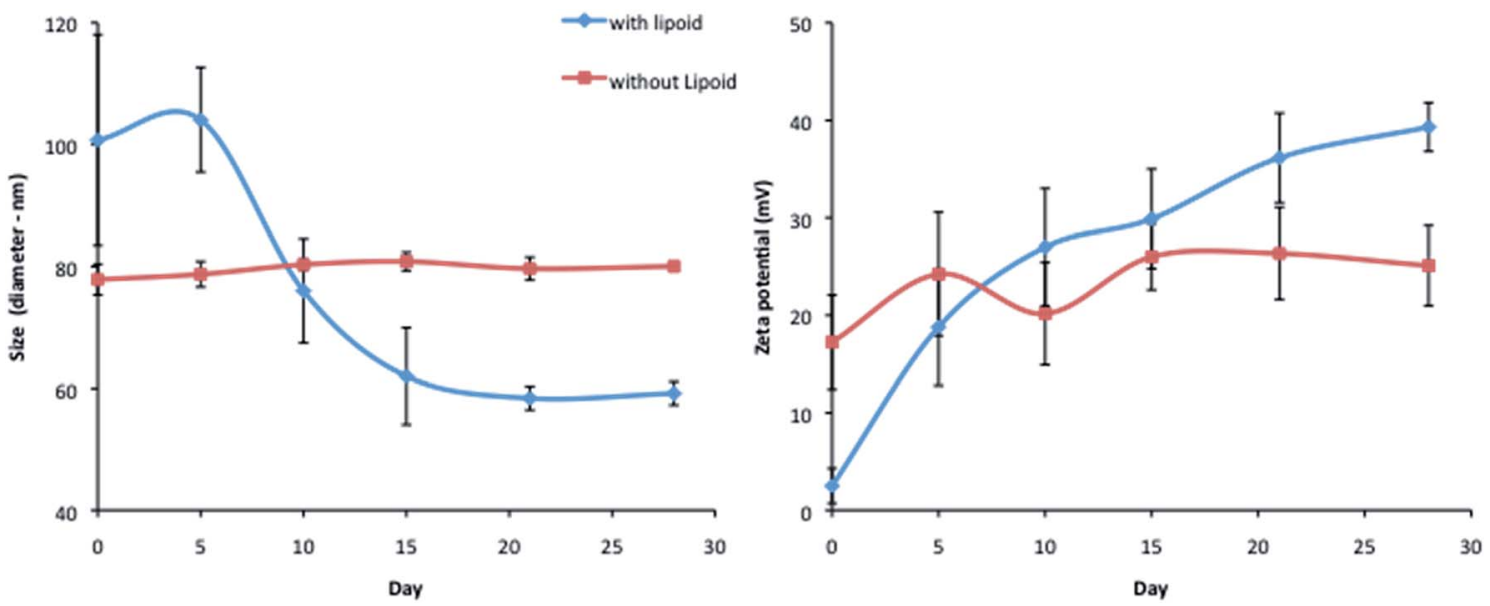

Fig. 2 Stability evaluation of siRNA LNCs with or without Lipoid®. Size (left) and zeta potential (right) were determined using DLS (Zetasizer, Malvern). siRNA LNCs with Lipoid® showed a time influence in terms of size and in zeta potential. At the opposite, long-term stability was observed for siRNA LNCs without Lipoid $®$, characterized by a diameter of $80 \mathrm{~nm}$ and a zeta potential of $+23 \mathrm{mV}$.

order to follow both LNC integrity and siRNA distribution, triple-labelled LNCs were formulated by loading them with the DiI/DiD FRET couple and with the siRNA-Alexa488 (see experimental section). With a $488 \mathrm{~nm}$ excitation, both Alexa488 fluorescence and FRET of DiI/DiD couple were simultaneously recorded (black spectrum in Fig. 3A: $I_{669} \mathrm{~nm} / I_{569} \mathrm{~nm}$ ratio 1.28 ). Once LNCs were completely disintegrated, for instance by dissolving them in organic solvents, the FRET was lost and the DiD emission band disappeared $\left(I_{669} \mathrm{~nm} / I_{569} \mathrm{~nm}\right.$ ratio decreased to 0.19 , data not shown).
Using the above considerations, spectral analysis allowed us to distinguish the intracellular situations corresponding to the three model spectra (Fig. 3A) and to generate the corresponding merged maps shown in pseudocolors (Fig. 3B): (i) blue corresponded to $75 \%$ of the initial FRET signal (high DiD signal); (ii) green corresponded to $44 \%$ of the FRET signal (high DiI signal), and (iii) red corresponded to free Alexa488-siRNAs, not colocalized with DiI/DiD ( $0 \%$ of FRET).

The results showed that after $0.5 \mathrm{~h}$ of incubation, LNCs were mostly observed in the cell membrane and only few of them
A

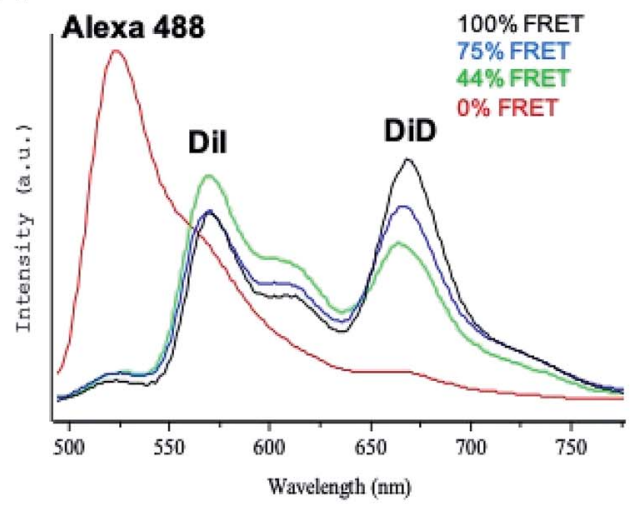

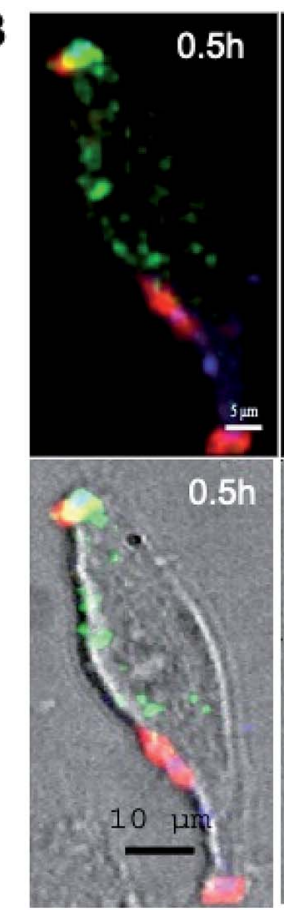

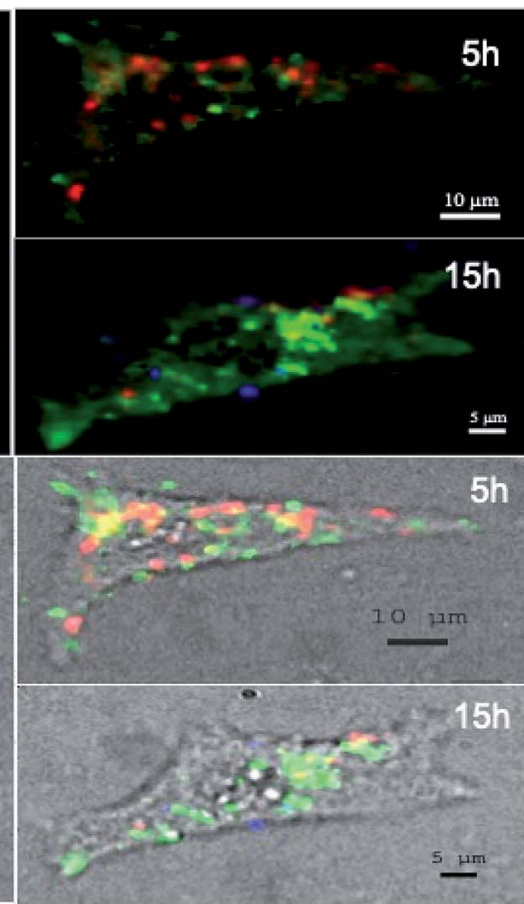

Fig. 3 Behavior of siRNA LNCs in SK-Mel28 cells over time, followed by fluorescence confocal spectral imaging, FCSI. (A) Model spectra of triplelabelled control LNCs (100\% of the FRET signal in black), partially degraded LNCs (75\% of the FRET signal in blue and $44 \%$ of the FRET signal in green) and Alexa 488-labeled siRNAs (0\% of the FRET signal in red). (B) Spectral maps (upper panel) and spectral maps merged with white light images of SK-Mel28 cells (lower panel) over time. The red color represents free siRNA (corresponding to the red spectrum) and the green and blue colors represent the degraded LNCs (corresponding to the green and blue spectra respectively). 
were found in the cytosol. Compared to the initial LNCs, a partial FRET decrease was observed, with a FRET signal of $75 \%$ for the LNCs in the cell membranes and of $44 \%$ in the cytosol (respectively blue and green spectra in Fig. 3A and blue and green zones in Fig. 3B). Red zones were observed in membranes, explained by free siRNA released from LNCs.

At $3.5 \mathrm{~h}$, the cell cytosol was significantly enriched with degraded LNCs (green zones). As a result of this degradation, a massive accumulation of free siRNA released from LNCs (large red zones) was observed in the cytosol (Fig. 3B). A fraction of LNCs (blue zones) was still observed mainly at the periphery of the cell membrane. At $5 \mathrm{~h}$, the siRNA was observed in numerous small zones in the cytosol. The dissociation of LNCs in cytosol led to rare blue zones, even on membranes. At $15 \mathrm{~h}$, only a few free siRNA (red spots) were observed in the cell, close to its periphery, while the DiI fluorescence (green) released from the degraded LNCs was still readily detected in the large zones of cytosol.

\section{Surface modified LNCs}

The formulation of siRNA loaded LNCs led to nanoparticles of $75 \mathrm{~nm}$ in diameter and characterized by a positive zeta potential
(+13 mV) (Table 1). The post-insertion of TE-PEG (Table 2) did not induce any modification. With DSPE-PEG, described in Table 2, at 5 and $10 \mathrm{mM}$ concentrations, the diameter increased gradually and the zeta potential became negative $(-10 \mathrm{mV}$; $-16 \mathrm{mV}$ respectively).

The presence of a maleimide group on DSPE-PEG led to the same nanoparticle diameter, but with a lower negative zeta potential ( $-9 \mathrm{mV}$ versus $-16 \mathrm{mV}$ ) (data not shown). The incorporation of fluorescent probes DiD, DiI or Alexa488 siRNA did not modify the physico-chemical properties of siRNA LNCs (data not shown).

The Affitin was grafted on $10 \mathrm{mM}$ malDSPE-PEG siRNA LNCs. Turbidity and protein detection confirmed the efficient grafting of the Affitins on DSPE-PEG LNC surface, as previously obtained with the grafting of an antibody by the same chemical reaction in Bourseau et al. ${ }^{31}$ The resulting formulation had a larger diameter than $10 \mathrm{mM}$ DSPE-PEG LNCs (103 and $94 \mathrm{~nm}$, respectively), leaving the other parameters unchanged (Table 1). All the different surface modified siRNA LNCs were monodispersed (PDI < 0.2) and the encapsulation efficiency of siRNA was evaluated around $35 \%$.

Table 1 Physico-chemical characterization and encapsulation yield of modified siRNA LNCs. LNCs are incubated with $10 \mathrm{mM}$ of tetraetherpolyethyleneglycol-2000 (TE-PEG) and 5 or $10 \mathrm{mM}$ of 1,2-distearoyl-sn-glycero-3-phosphoethanolamine- $\mathrm{N}$-[methoxy(polyethyleneglycol)2000] (DSPE-PEG). Grafting of the Affitins $(110 \mu \mathrm{M})$ is performed on $10 \mathrm{mM}$ maleimideDSPE-PEG post-inserted LNCs ${ }^{a}$

\begin{tabular}{|c|c|c|c|c|}
\hline Formulations & Diameter (nm) & PDI & Zeta potential (mV) & $\mathrm{EE}(\%)$ \\
\hline LNCs & $74 \pm 4$ & $0.05 \pm 0.01$ & $+13 \pm 6$ & $35 \pm 5$ \\
\hline TE-PEG LNCs & $74 \pm 3$ & $0.074 \pm 0.04$ & $+15 \pm 1$ & $30 \pm 5$ \\
\hline 5 mM DSPE-PEG LNCs & $85 \pm 2$ & $0.13 \pm 0.05$ & $-10 \pm 4$ & $33 \pm 4$ \\
\hline 10 mM DSPE-PEG LNCs & $94 \pm 14$ & $0.16 \pm 0.08$ & $-16 \pm 4$ & $36 \pm 7$ \\
\hline 10 mM DSPE-PEG-Affitins LNCs & $103 \pm 13$ & $0.16 \pm 0.02$ & $-16 \pm 4$ & $36 \pm 3$ \\
\hline
\end{tabular}

${ }^{a}$ Results $(n=3)$ are expressed as mean measure \pm standard deviation..$^{29}$ No difference was demonstrated on encapsulation efficiency ${ }^{30}$ using ANOVA $1 \mathrm{~W}$, post hoc Tukey.

Table 2 Structure of TE-PEG and DSPE-PEG used for siRNA LNCs post-insertion. All polyethylene glycol (PEG) polymer, (tetraether[methoxy(polyethyleneglycol)-2000] (TE-PEG), 1,2-distearoyl-sn-glycero-3-phospho ethanolamine- $\mathrm{N}$-[methoxy(polyethyleneglycol)-2000] (DSPE-PEG)) and 1,2-distearoyl-sn-glycero-3-phosphoethanolamine- $N$-[maleimide(polyethyleneglycol)-2000] (malDSPE-PEG) have same PEG length evaluated at $2000 \mathrm{kDa}$

Polymer Structure PEG length (kDa)

TE-PEG

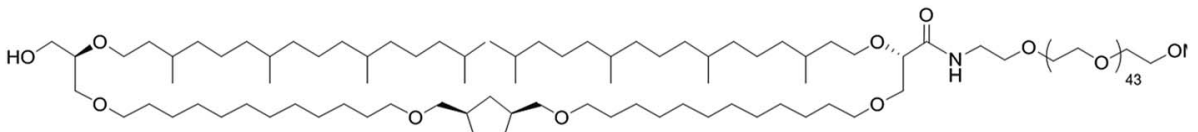

DSPE-PEG

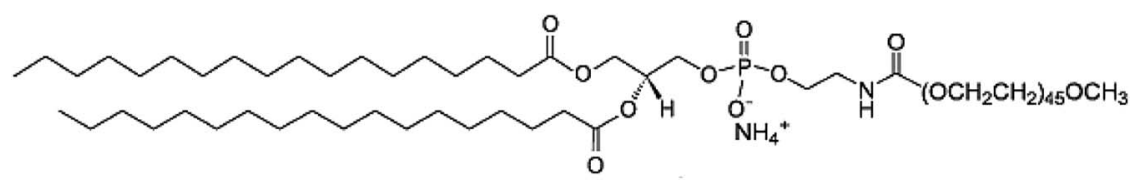

malDSPE-PEG

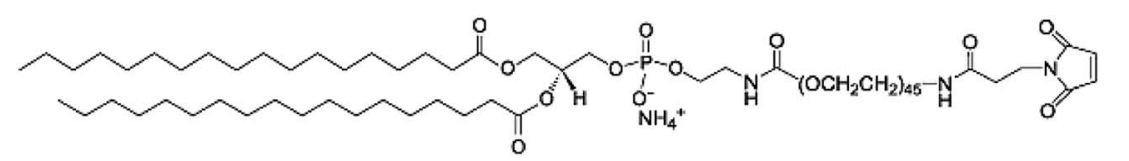




\section{Cellular uptake into melanoma cells}

The internalization of LNCs and siRNA was evaluated by fluorescence microscopy and flow cytometry, after surface modification by different PEG (5 mM DSPE-PEG, 10 mM DSPE-PEG, $10 \mathrm{mM}$ TE-PEG, $10 \mathrm{mM}$ DSPE-PEG-Affitins) (Fig. 4 and 5). After $48 \mathrm{~h}$ of incubation, TE-PEG LNCs were observed in SK-
Mel28 cells via the Alexa488 and DiD fluorescences. On the opposite, no signal was detected after incubation with the other formulations (5, $10 \mathrm{mM}$ and DSPE-PEG-Affitins) (Fig. 4). To quantify the proportion of positive cells, flow cytometry was performed after quenching of Alexa488 with trypan blue to discriminate the adsorbed from internalized siRNA (Fig. 5). The analysis confirmed the large proportion of positive cells after

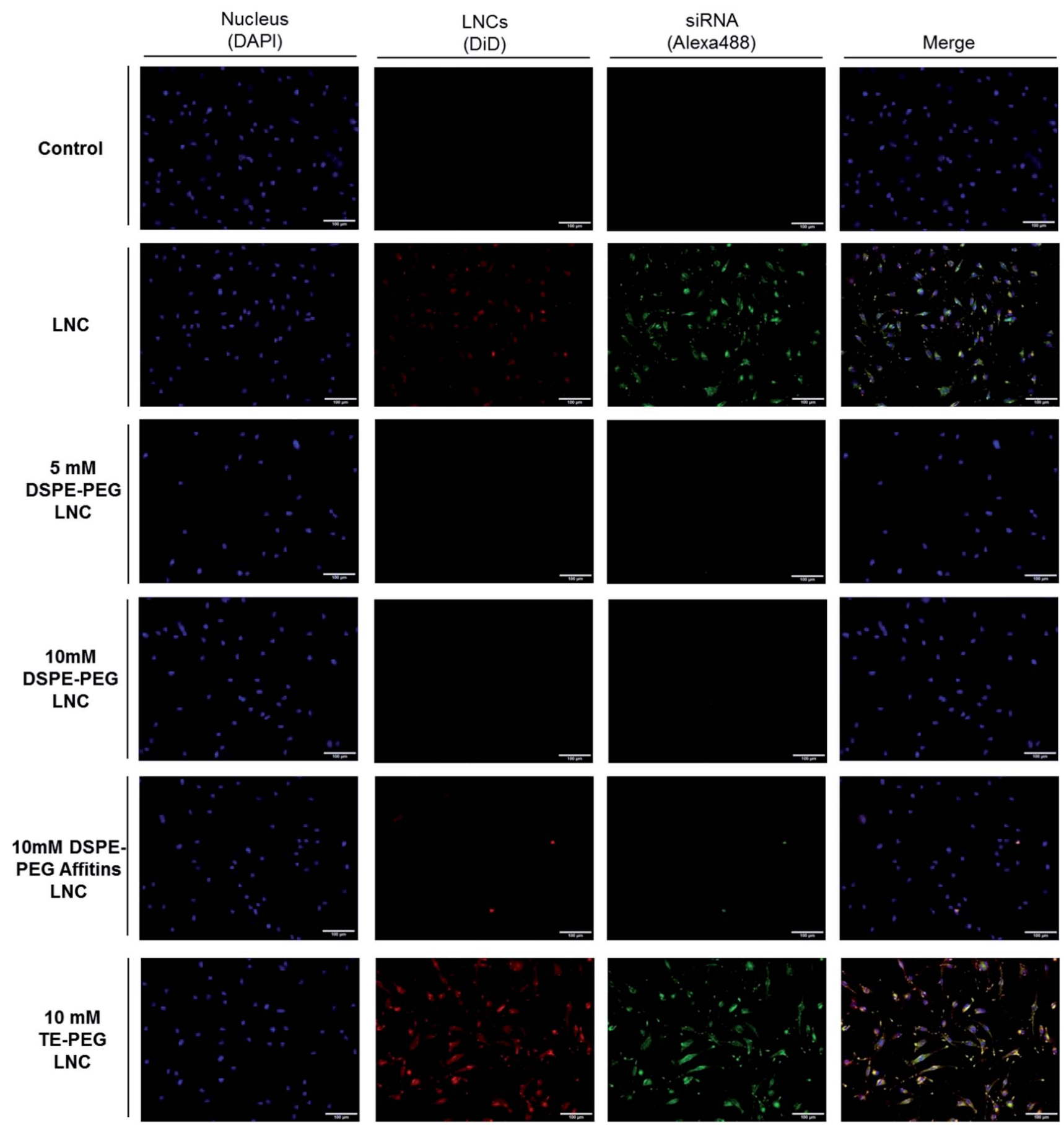

Fig. 4 Cell uptake of siRNA LNCs in melanoma cells according to the surface modification. After two days of incubation, fluorescence microscopy was performed with siRNA LNCs on SK-Mel28 human melanoma cells. Cells are fixed on Labtek slide and nucleus staining was performed with DAPI (blue). Double fluorescent probes are used to follow siRNA LNCs: lipophilic DiD (red) and Alexa488 siRNA (green). Analysis confirmed the internalization of siRNA LNCs without surface modification or after post-insertion of TE-PEG. On the opposite, DSPE-PEG LNCs showed no fluorescent signal. White scale bar $=100 \mu \mathrm{m}$. 
A
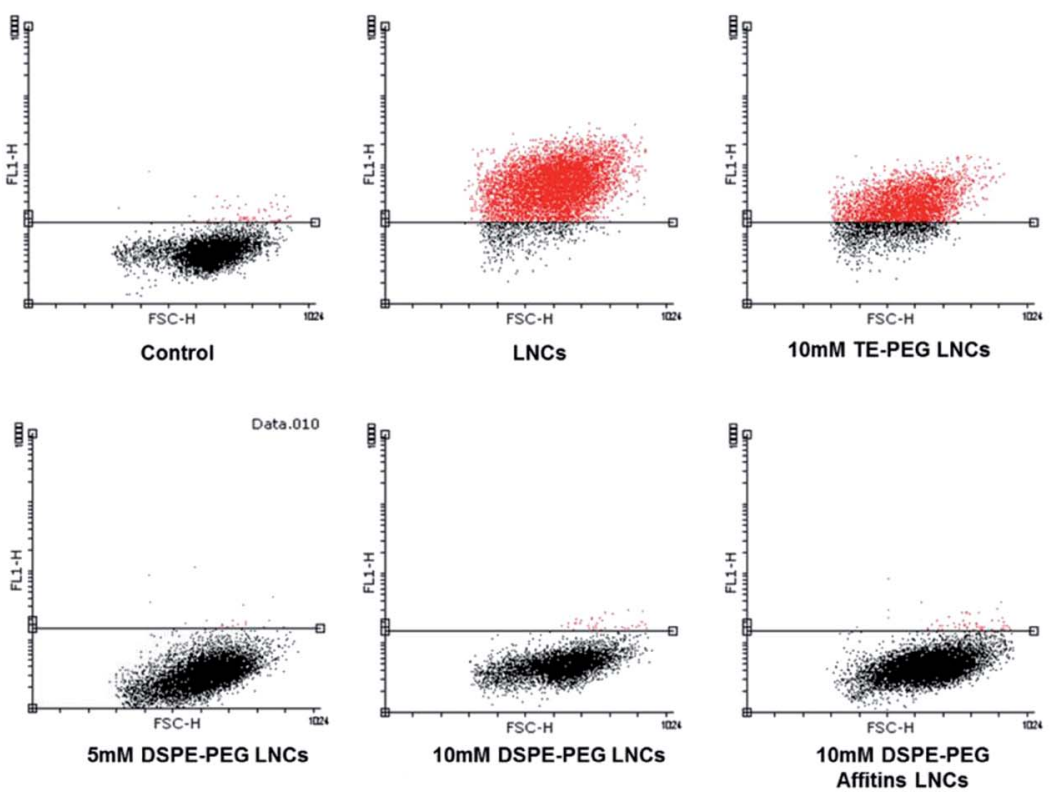

B

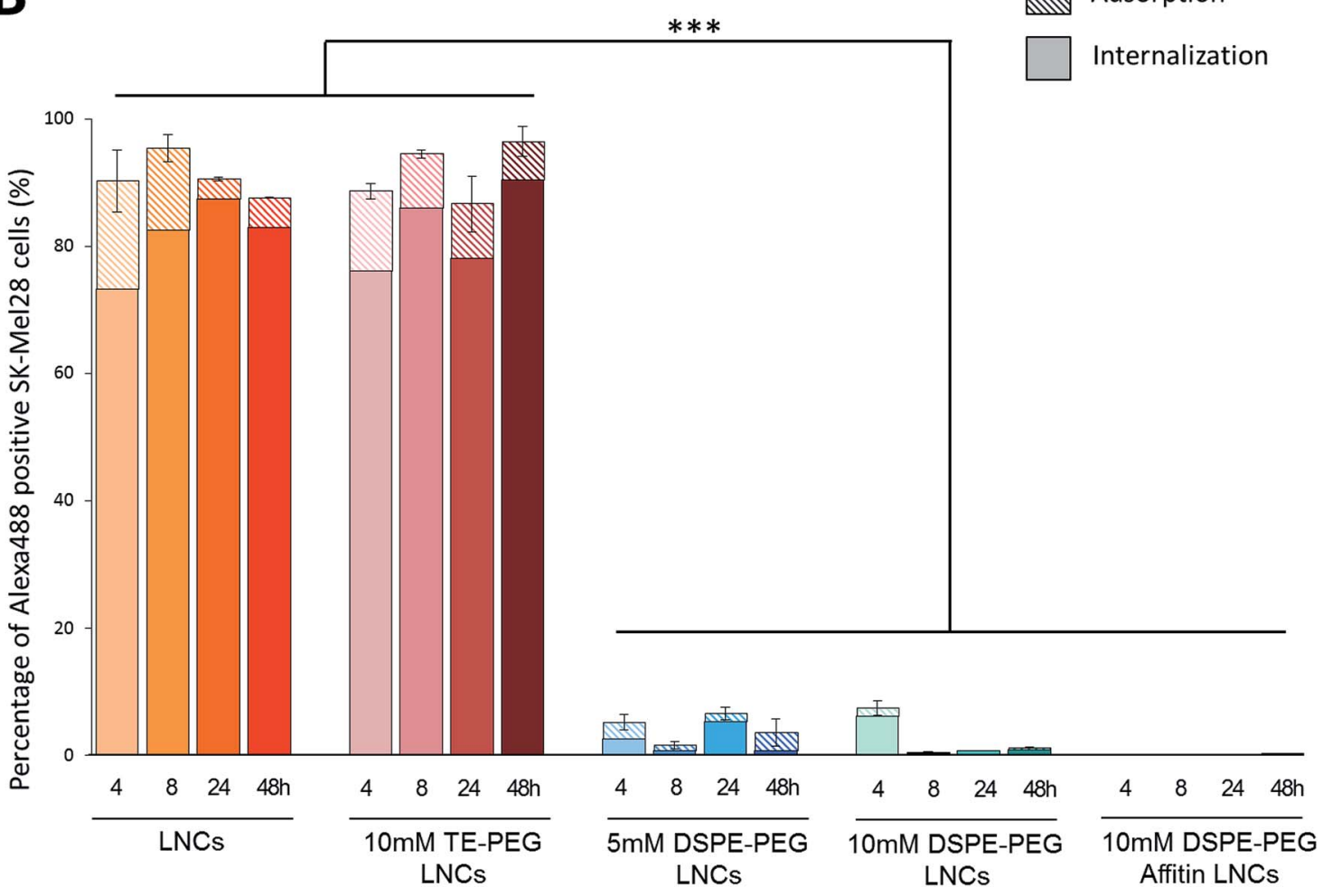

Fig. 5 Evaluation of siRNA positive cells by flow cytometry analysis over time. (A) Dot plot analysis evidenced the large uptake of Alexa488 siRNA into SK-Mel28 cells for LNCs and TE-PEG LNCs. (B) Histograms showed the difference between internalized and adsorbed (hatched part of bar) siRNA, after trypan blue quenching. Data confirmed the large proportion of positive cells in LNCs and TE-PEG LNCs, with a low adsorption phenomenon $(<10 \%)$, contrary to 5 and $10 \mathrm{mM}$ DSPE-PEG LNCs and $10 \mathrm{mM}$ DSPE-PEG-Affitins LNCs at $48 \mathrm{~h}$. The mean percentage of fluorescent cells was compared to control cells without any fluorescence \pm standard error of mean SEM $(n=3)$. Statistical analysis was performed with ANOVA 1W, post hoc Tukey, ***p $=0.001$

incubation with non-modified and TE-PEG post-inserted LNCs (Fig. 5B). After $4 \mathrm{~h}, 90 \%$ of cells were Alexa488 positive with nonmodified and TE-PEG LNCs and the percentage was constant until $48 \mathrm{~h}$. In this case, the proportion of absorbed siRNA was limited to $20 \%$ at $4 \mathrm{~h}$ and decreased over time to a few percent (Fig. 5B). For DSPE-PEG LNCs, flow cytometry proved the 


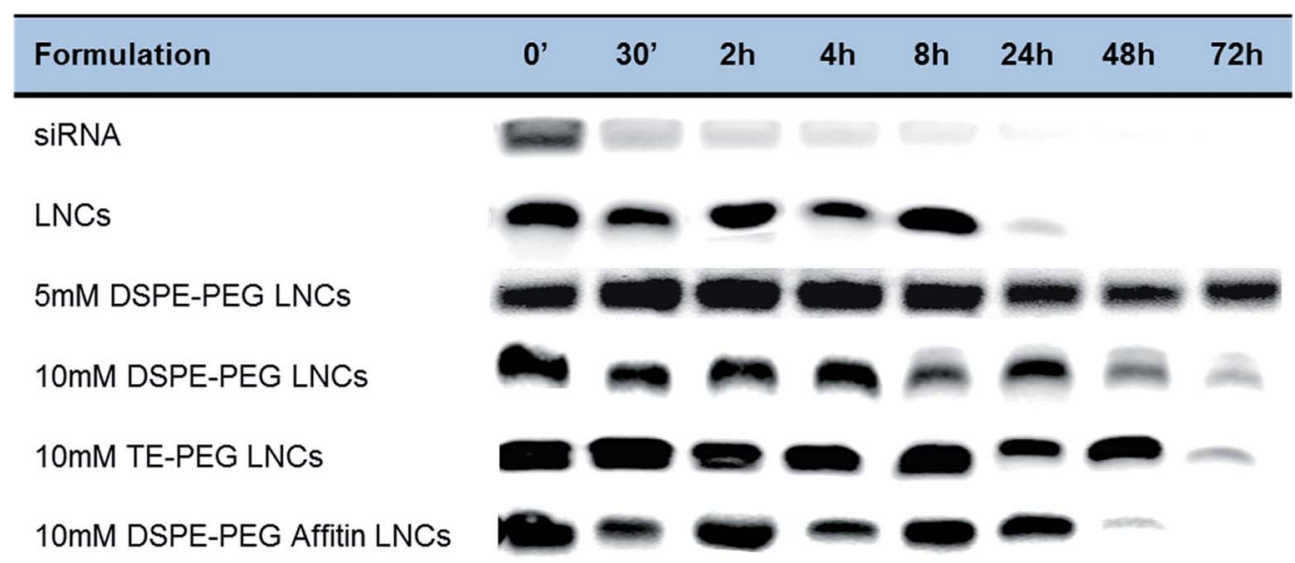

Fig. 6 Evaluation of plasmatic protection of siRNA LNCs with different surface modifications. UV light detection of siRNA (thanks to ethidium bromide electrophoresis) after incubation with human plasma at $37^{\circ} \mathrm{C}$ showed the efficient nuclease protection of siRNA by pegylated LNCs.

significant lower percentage of internalized cells by siRNA (lower than 10\%). Moreover, compared to $10 \mathrm{mM}$ DSPE-PEG LNCs, the presence of Affitin did not modify the behavior of siRNA LNCs on melanoma cells.

\section{Plasma protection}

The siRNA protection was evaluated after incubation with human plasma, at physiological temperature (Fig. 6). In control conditions with siRNA alone, a fast degradation by plasmatic nucleases was observed after $30 \mathrm{~min}$. LNCs demonstrated their efficacy to protect the siRNA against nucleases for at less $8 \mathrm{~h}$, and longer protection times up to $24 \mathrm{~h}$ or $48 \mathrm{~h}$ were observed for all PEGylated LNCs (TE-PEG, 5 and $10 \mathrm{mM}$ DSPE-PEG). The binding of Affitin to DSPE-PEG LNCs did not modify the protection effect against nucleases up to $24 \mathrm{~h}$.

\section{Biodistribution on healthy mice}

Biofluorescence imaging on animals was performed to evaluate the biodistribution of DiD siRNA LNCs with or without surface modification, after i.v. injection in healthy mice (Fig. 7). For non-modified siRNA LNC, the maximal signal was obtained after $1 \mathrm{~h}$ injection and induced a strong hepatic accumulation after $3 \mathrm{~h}$ and until $8 \mathrm{~h}$ (Fig. 7A). The $5 \mathrm{mM}$ DSPE-PEG postinserted LNCs showed an important liver localization between $5 \mathrm{~h}$ and $24 \mathrm{~h}$ with a maximum at $8 \mathrm{~h}$. The highest concentration of DSPE-PEG $(10 \mathrm{mM})$ on LNCs seemed to limit liver accumulation: the fluorescence signal was diffused (Fig. 7A). However, after $48 \mathrm{~h}$, organ fluorescence evidenced the high amount of 5 and $10 \mathrm{mM}$ DSPE-PEG LNCs, especially in liver, spleen, kidneys and bladder (Fig. 7B). Surprisingly, a very high fluorescence signal was observed in ovary with 5 and 10 mM DSPE-PEG LNCs. The Affitin grafting modified slightly the biodistribution, with a liver accumulation between $1 \mathrm{~h}$ and $24 \mathrm{~h}$ (Fig. 7A). However, the global fluorescence signal of DSPE-PEG-Affitin LNCs showed no important retention in undesirable organs (Fig. 7B). Concerning the TE-PEG siRNA LNCs, biofluorescence imaging and organ dissection demonstrated the accumulation into liver over time with a higher signal at $48 \mathrm{~h}$ (Fig. 7). To conclude, the analysis of the organs confirmed the high level of fluorescence into different organs such as spleen, kidney, lung, ovary, heart or bladder for all pegylated LNCs (5 mM, 10 mM DSPE-PEG and TE-PEG LNCs) (Fig. 7B).

\section{Biodistribution on subcutaneous melanoma mice model}

Biofluorescence imaging was performed on subcutaneous melanoma mice model (Fig. 8). In order to better visualize the tumor, animals were put on the left flank. A diffuse distribution of fluorescence with a liver accumulation was observed for unmodified LNCs. No specific fluorescence signal was colocalized with the tumor site, confirming the important hepatic accumulation (Fig. 8B). Biofluorescence imaging of DSPE-PEG LNCs showed a fast tumor accumulation (after $1 \mathrm{~h}$ and $5 \mathrm{~h}$, for $10 \mathrm{mM}$ and $5 \mathrm{mM}$ DSPE-PEG LNCs respectively). This passive accumulation of siRNA LNCs modified by DSPEPEG was confirmed by tumor fluorescence at $48 \mathrm{~h}$ (Fig. 8). Concerning DSPE-PEG-Affitin LNCs, no signal was observed in the tumor site, demonstrating a large elimination of this formulation after $48 \mathrm{~h}$ compared to the other formulations (Fig. 8B). Finally, LNCs modified with TE-PEG showed a persistent tumor localization $24 \mathrm{~h}$ after intravenous injection (Fig. 8A). Interestingly, at $48 \mathrm{~h}$, TE-PEG LNCs showed the highest fluorescence signal in tumor compared to unmodified and DSPE-PEG-Affitin LNCs (Fig. 8B).

\section{Discussion}

LNCs have been developed 20 years ago: the process is based on inversion phase of emulsion by mixing phospholipids, triglycerides, tensio-active polymers, salt and water. ${ }^{32}$ Firstly used for the encapsulation of lipophilic compounds, such as paclitaxel, ${ }^{33,34}$ LNCs were adapted more recently for the encapsulation of hydrophilic moieties via lipoplexes (DNA, siRNA). ${ }^{35}$ However, stability over time was not satisfying, especially in terms of size, such as described in our previous works. ${ }^{9}$ Some experiments led to the hypothesis of a saturated LNC surface, induced by the large amount of phospholipids, explaining the low stability of the system. This optimization of the process was 

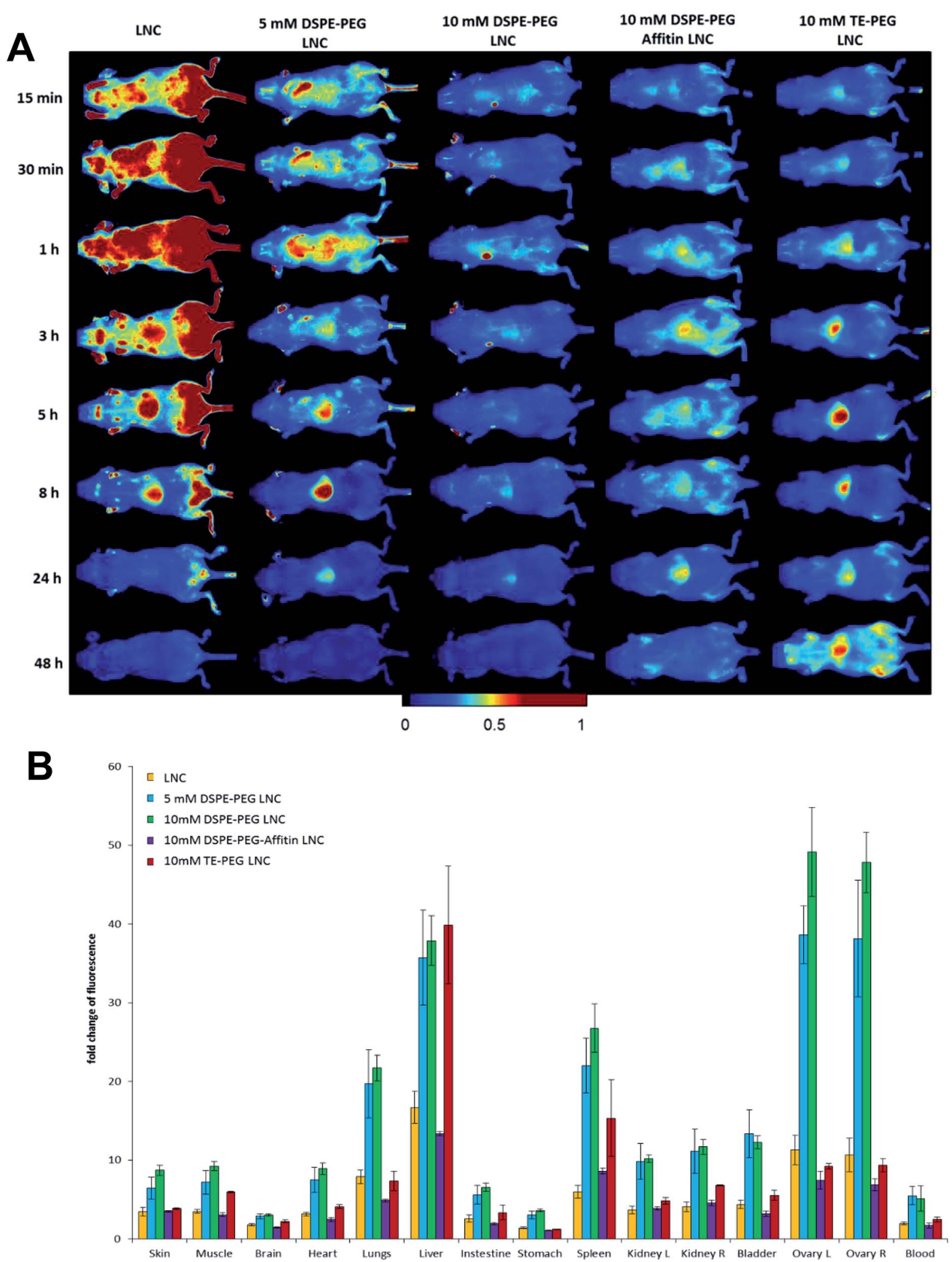

Fig. 7 Evaluation of siRNA LNC biodistribution after intravenous injection in healthy NRMI nude female mice. (A) LNCS (yellow), 5 mM DSPE-PEG LNCs (blue), 10 mM DSPE-PEG LNCs (green), 10 mM DSPE-PEG-Affitin LNCs (purple) and 10 mM TE-PEG LNCs (red), loaded by a fluorescent tracer, DiD, were injected intravenously in healthy mice $(n=4)$. In vivo biofluorescence imaging (BFI) was evaluated at different times (15 min, $30 \mathrm{~min}, 1,3,5,8,24$ and 48 hours after injection) from decubitus dorsal view, to follow biodistribution. (B) Two days after injection, biodistribution in organs was evaluated, proving the high liver accumulation for pegylated LNCs compared to LNCs and 10 mM DSPE-PEG-Affitins LNCs that were fully eliminated. Mean fluorescence \pm standard error of the mean (SEM). 

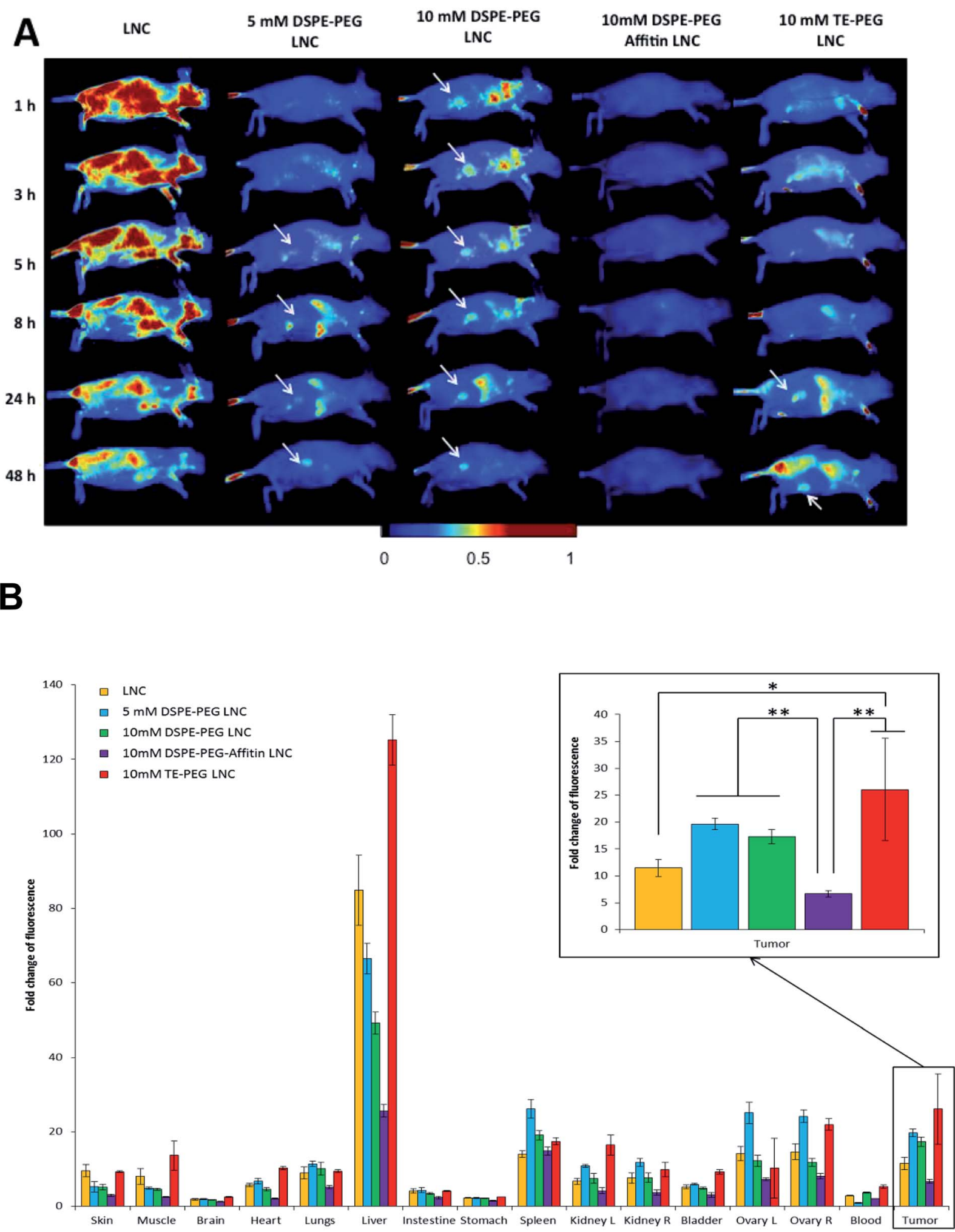

Fig. 8 Evaluation of siRNA LNC biodistribution after intravenous injection in subcutaneous tumor bearing NRMI nude female mice. (A) LNCS (yellow), 5 mM DSPE-PEG LNCs (blue), 10 mM DSPE-PEG LNCs (green), 10 mM DSPE-PEG-Affitin LNCs (purple) and 10 mM TE-PEG LNCs (red), loaded by a fluorescent tracer, DiD, were injected intravenously in mice $(n=4)$ and vivo biofluorescence imaging (BFI) were taken at different times (1, 8, 24 and 48 hours after injection) from lateral views to follow their biodistribution and the potential tumor targeting. BFI revealed the specific tumor targeting in subcutaneous melanoma model for pegylated LNCs over time. (B) Two days after injection; biodistribution in organs proved the high liver accumulation for LNCs and TE-PEG LNCs compared to DSPE-PEG LNCs and 10 mM DSPE-PEG-Affitins LNCs. Fluorescence analysis in tumor demonstrated the best result for TE-PEG and DSPE-PEG LNCs. Mean fluorescence \pm standard error of the mean (SEM), ANOVA 1W, post hoc Tukey, ${ }^{*} p<0.05$ and ${ }^{* *} p<0.01$. 
assessed by the elimination of the lecithin (Fig. 1) (patent: FR 4185991, 24 Sept 2014).

The siRNA loaded LNC cell uptake was studied overtime on living melanoma cells, by fluorescence confocal multispectral imaging (FCSI). The loss of FRET signal could correlate with a nanocapsule degradation. After a short incubation time $(0.5 \mathrm{~h})$ at $37^{\circ} \mathrm{C}$, a part of the siRNA LNCs seemed to be degraded before or by the contact with the cell membrane, explained by a decrease of the DiD component from the FRET signal. This element may be due to a fusion of LNCs with the cell membrane, supported by DOTAP/DOPE lipids, and leading to the efficient cytoplasm delivery of siRNAs. Indeed, localization of fluorescence was diffused into the cytoplasm: concentrated spots could be observed after endocytosis phenomenon such as with calcium phosphate particles developed notably by Xie et al. ${ }^{36}$ siRNA transfection seemed to reach a maximum at $3.5 \mathrm{~h}$, where the siRNAs were stained over large cytosolic regions. At $5 \mathrm{~h}$, the siRNAs seemed to be dispatched in the whole cell and the fluorescence signal decreased clearly at longer incubation time ( $15 \mathrm{~h})$, indicating a siRNA release. The same intracellular trafficking and kinetic were observed for calcium phosphate particles with a maximal cytoplasmic loading after $4 \mathrm{~h}$ of incubation. ${ }^{36}$ Furthermore, the cellular trafficking of blank LNCs, without any drug, was investigated by Paillard et $a l^{37}$ Interestingly, results clearly showed blank LNCs internalized through an energy dependent mechanism such as clathrin- or caveolin-mediated endocytosis pathways. The presence of new excipients in siRNA LNC formulation, cationic DOTAP and zwitterionic DOPE lipids ${ }^{6}$ would allow additional fusogenic properties to the nanosystem: indeed DOPE is well known to undergo transition from bilayer to inverted hexagonal structures at low $\mathrm{pH}$ known to catalyze the fusion process with the endosomal membrane. ${ }^{38,39}$

The surface modification of nanomedicines with innovative polymers represents an important challenge to improve tumor targeting after intravenous injection. In this work, various surface coatings (DSPE-PEG, TE-PEG and Affitin protein grafting) were applied to siRNA LNCs and validated without siRNA loading modification. In order to control the fluorescence and avoid variation between the different formulations, all biofluorescent imaging experiments were performed using one batch of fluorescent LNCs. This precaution taken, the altered biodistribution of PEG LNCs, compared to unmodified LNCs, could not be justified by a different fluorescence intensity between formulations. The biodistribution pattern obtained for PEG LNCs showed weak variations, excepted for the liver, as already observed in a previous study. ${ }^{40}$ Supplementary blood analysis confirmed the presence of PEG LNCs, $1 \mathrm{~h}$ and $3 \mathrm{~h}$ after intravenous injection, such as for DNA LNCs. ${ }^{12}$ This result could be explained by a difference of diffusion into the blood circulation, with a lower peripherical distribution. ${ }^{40}$ However, the addition of DSPE-PEG induced considerable limitation of in vitro cell uptake, avoiding intracellular siRNA delivery. This result could be explained by a steric hindrance between grafted chains and cell membrane according to PEG concentration, length and conformation. ${ }^{41-43}$ The comparison of two PEG concentrations, in biodistribution and tumor targeting studies, evidenced the advantage of choosing the lowest tested concentration of PEG. A theoretical calculation estimated that $5 \mathrm{mM}$ led to a surface coating of 10 molecules per $\mathrm{nm}^{2}$ or approximatively 3000 PEG molecules per LNC. ${ }^{12}$

The grafting of Affitins on the LNC surface, a small artificial model binding protein, modified the behavior of LNCs as already observed for nanomedicines modified with peptides. ${ }^{41,44}$ As a first step toward active targeting with LNCs, we wanted to study if an Affitin model, i.e. without any affinity for a melanoma biomarker, had intrinsic properties compatible with our goals. In this work, the Affitin did not alter the plasma protection of siRNA in $10 \mathrm{mM}$ DSPE-PEG LNCs. Moreover, Affitin grafted on LNCs was associated with the lowest fluorescence signal $48 \mathrm{~h}$ after injection, confirming the rapid elimination and the absence of accumulation in undesirable organs in healthy and melanoma bearing mice. The $10 \mathrm{mM}$ DSPE-PEG-Affitin LNCs induced, at early times, a higher hepatic accumulation than for the $10 \mathrm{mM}$ DSPE-PEG LNCs, probably due to the reduced stealth effect of the PEG with the covalent peptide grafting or/and due to a potential recognition of the peptide by the kupffer cells.

The $10 \mathrm{mM}$ DSPE-PEG-Affitin LNC showed the lowest fluorescence signal in tumor site: even if the Affitin model used had no specificity for a melanoma biomarker, a passive targeting was at least expected. A study of Rangger et al., showed the necessity to study the balance between pegylation and ligand grafting to achieve an efficient and optimized targeting. ${ }^{41}$ Indeed, in our study, the Affitin conjugation to the PEG moiety significantly reduced the in vivo tumor uptake compared with pegylated LNC: the tumor accumulation was in fact comparable to LNC without any surface modification. The Affitin model grafting probably prevented the stealth effect of PEG, reducing thus the passive accumulation. In conclusion, these results highlight one of the most challenging problems in the delivery of nanomedicines: developing high-quality formulations that are fully characterized and with a specific accumulation of nanoparticles in tumor. ${ }^{45}$

Finally, the tetraether-PEG (TE-PEG) appears as an innovative solution to replace DSPE-PEG, with a low influence on cell uptake and a good plasma protection compared to nonmodified LNCs. This archaeal lipid is known to improve the stability of liposomes. ${ }^{17}$ Interestingly, TE-PEG LNCs had a short half-life time in blood, but the biodistribution profile evidenced a higher tumor accumulation overtime. This efficacy could be explained by a different PEG conformation on the LNC surface. Beyond classic surface modifications of nanomedicines providing longevity in blood stream, some efforts have been performed to develop "smart" nanocarriers, ${ }^{18,46}$ via a stimulisensitive drug delivery system in response to various internal stimuli such as $\mathrm{pH}$, temperature, or external signals, as magnetism or ultrasounds. ${ }^{18}$ This TE-PEG appears as a good candidate to create a "smart" targeting with a $\mathrm{pH}$-sensitive link, adapted to the melanoma acidic microenvironment. ${ }^{16,47}$ Moreover, Affitins display a high stability for acidic $\mathrm{pH},{ }^{20}$ and future works should test the efficiency of TE-PEG-Affitin formulations using a specific melanoma biomarker Affitin. 
The challenges and barriers to the systemic delivery of siRNA are numerous such as specificity, degradation, penetration of the cell membrane and endosomal escaping. Various natures of nanoparticles have been studied for siRNA delivery such mesoporous silica nanoparticles, ${ }^{\mathbf{4 8}}$ gold nanoparticles, ${ }^{\mathbf{4 9}}$ carbon nanotubes, ${ }^{50}$ polymeric nanoparticles ${ }^{51}$ or lipid-based nanoparticles ${ }^{52}$ with respective disadvantages: in vivo toxicity, poor stability, limited biocompatibility, high cost of large scale production. In this study, lipid nanocapsules have been used, demonstrating a good stability over time, a plasma protection, an impressive cell internalization, with a release of siRNA into the cytosol. Moreover, in this work, the versatility of the surface functionalization has been demonstrated with a significant tumor accumulation for the TE-PEG LNCs.

\section{Experimental}

\section{SiRNA LNC formulation}

Blank LNCs. LNCs were formulated as described previously, ${ }^{32}$ by combining 20.5\% w/w Labrafac WL 1349 (capryliccapric acid triglycerides, Gatefossé S.A. Saint-Priest, France), 1.5\% w/w Lipoid S75-3 (Lipoid GmbH, Ludwigshafen, Germany), $16.9 \%$ w/w Kolliphor ${ }^{\circledR}$ HS 15 (BASF, Ludwigshafen, Germany), $1.8 \%$ w/w NaCl (Prolabo, Fontenay-sous-Bois, France), and $59.8 \% \mathrm{w} / \mathrm{w}$ water (obtained from a Milli-Q system, Millipore, Paris, France) with magnetic stirring. Three temperature cycles between 60 and $95{ }^{\circ} \mathrm{C}$ were performed to obtain phase inversions of the emulsion. Subsequent rapid cooling and dilution with ice-cooled water at the last phase inversion temperature (PIT) led to formation of blank LNCs.

Lipoplexes and siRNA LNCs. For siRNA encapsulation, the nucleic acids were firstly complexed with DOTAP/DOPE lipids. Briefly, the cationic lipid DOTAP (Avanti ${ }^{\circledR}$ Polar Lipids Inc., Alabaster, AL, USA), solubilized in chloroform, was weighted at the ratio 1/1 (mol/mol) with the neutral lipid DOPE (Avanti ${ }^{\circledR}$ Polar Lipids Inc., Alabaster, AL, USA) to obtain a final concentration of $30 \mathrm{mM}$ of cationic lipid charges, based on the number of lipid charges per molecule, i.e., 1 for DOTAP. After evaporation of chloroform under vacuum, deionized water was added to rehydrate the DOTAP/DOPE lipid film overnight at $4{ }^{\circ} \mathrm{C}$. The lipid film was then sonicated for $30 \mathrm{~min}$. Lipoplexes were formulated as a simple equivolume mix of siRNA and DOTAP/ DOPE lipids. In this study, the $\mathrm{Na} / \mathrm{K}$ ATPase alpha 1 subunit siRNA (sense sequence: 5'-GGGCAGUGUUUCAGGCUAAdTdT3'; antisens: 5'-UUAGCCUGAAACACUGCCCdTdT-3'; Eurogentec, Seraing, Belgium) was used.

The complexes were characterized by the charge ratio (CR), i.e., the ratio between the positive charges of the lipids and negative charges of the nucleic acids ( \pm ratio), fixed to 5 considering our previous results. ${ }^{\mathbf{1 0}}$ These pre-formulated lipoplexes were added during the last phase inversion temperature (PIT) of LNC formulation leading to the formation of siRNA LNCs.

Optimization of the formulation and stability of siRNA LNCs. Regarding our previous characterization of siRNA LNCs, a tensiometry study evidenced the interfacial position of lipoplexes in the formulation of siRNA LNCs. ${ }^{9}$ The low stability could be explained by a saturation of tensioactive molecules at LNC surface. In this way, formulations of siRNA LNCs were assessed with and without Lipoid® (lecithin). The stability of the different formulations in terms of size and zeta potential was evaluated by dynamic light scattering as described in the experimental section.

Fluorescent siRNA LNCs. To formulate fluorescent siRNA LNCs, a solution of DiD (1,1'-dioctadecyl-3,3,3',3'-tetramethylindodicarbocyanine perchlorate; $\lambda_{\mathrm{ex}}=644 \mathrm{~nm} ; \lambda_{\mathrm{em}}=665 \mathrm{~nm}$ ) and DiI (1,1'-dioctadecyl-3,3,3', $3^{\prime}$-tetramethylindocarbocyanine perchlorate; $\lambda_{\mathrm{ex}}=549 \mathrm{~nm} ; \lambda_{\mathrm{em}}=565 \mathrm{~nm}$ ) (Invitrogen, CergyPontoise, France) solubilized in acetone at $25 \mathrm{mg} \mathrm{mL}^{-1}$ was prepared.

For in vitro experiments, the DiD concentration was fixed at $200 \mu \mathrm{g} \mathrm{mL}^{-1}$ of LNC suspension or corresponding to $1.36 \mathrm{mg}$ of DiD per grams of Labrafac ${ }^{\circ}$. Regarding the FRET experiments, the DiD concentration was unchanged and the ratio DiD/DiI was fixed at $1 / 2(\mathrm{~mol} / \mathrm{mol})$, corresponding to a DiD concentration at $400 \mu \mathrm{g} \mathrm{mL} \mathrm{m}^{-1}$ of LNC suspension. For biodistribution on animals, the DiD concentration was fixed at $33 \mu \mathrm{g} \mathrm{mL}{ }^{-1}$ of LNC suspension or $0.23 \mu \mathrm{g} \mathrm{mL}^{-1}$ of Labrafac.

The adequate volume of $\mathrm{DiD}$ or DiD/DiI solubilized in acetone was incorporated in Labrafac ${ }^{\circledR}$ and acetone was evaporated at room temperature. The formulation process was unchanged, and formulation was stored at $4{ }^{\circ} \mathrm{C}$, protected from light. For siRNA fluorescent LNCs, a fluorescent Alexa488 siRNA $\left(\lambda_{\mathrm{ex}}=488 \mathrm{~nm} ; \lambda_{\mathrm{em}}=524 \mathrm{~nm}\right.$, Eurogentec) was used.

\section{Purification and post-insertion of PEG polymers}

Purifications were performed in water (obtained from a Milli-Q system, Millipore, Paris, France) with PD10 Sephadex column (Amersham Biosciences Europe, Orsay, France) to remove the non-encapsulated siRNA and lipoplexes. ${ }^{9}$ At the end, the $\mathrm{NaCl}$ concentration was adjusted to physiological concentration (150 $\mathrm{mM})$.

The polymers used for post-insertion were 1,2-distearoyl-snglycero-3-phosphoethanolamine- $N$-[methoxy(polyethyleneglycol)2000] (DSPE-PEG) (mean molecular weight $\mathrm{MM}_{\mathrm{w}}=2805 \mathrm{~g}$ $\left.\mathrm{mol}^{-1}\right), \quad$ 1,2-distearoyl-sn-glycero-3-phosphoethanolamine- $N$ [maleimide(polyethyleneglycol)-2000] (malDSPE-PEG) (mean molecular weight $\mathrm{MM}_{\mathrm{w}}=2942 \mathrm{~g} \mathrm{~mol}^{-1}$ ) (Avanti Polar Lipids, Inc, Alabaster, USA) and tetraether-[methoxy(polyethyleneglycol)2000] (TE-PEG) $\left(\mathrm{MM}_{\mathrm{w}}=3217 \mathrm{~g} \mathrm{~mol}^{-1}\right)$ synthesized according to Barbeau et al. $^{17}$ Polymers and siRNA LNCs were co-incubated $4 \mathrm{~h}$ at $37^{\circ} \mathrm{C}$ with a final polymer concentration adjusted to 5 or $10 \mathrm{mM}$.

\section{Affitin coupling on DSPE-PEG LNCs}

The Affitin H4 used in this study is specific to hen egg white lysozyme, and this "model" Affitin has been described previously. ${ }^{21}$ To allow the covalent coupling via thiol/maleimide chemistry, a cysteine residue was added to C-terminus of this Affitin by directed mutagenesis. The resulting Affitin (H4-Ct) was coupled to LNCs according to Bourseau et al. ${ }^{\mathbf{3 1}}$ Briefly, a solution at $1 \mathrm{mg} \mathrm{mL} \mathrm{mL}^{-1}$ (corresponding to $110 \mu \mathrm{M}$ ) of Affitin was prepared in water and mixed with DTT (dithiothreitol, 
Sigma-Aldrich), a strong reducing agent, at $0.012 \mathrm{mg} \mathrm{mL}^{-1}$ (corresponding to $80 \mu \mathrm{M}$ ) under stirring at room temperature during $1 \mathrm{~h}$. Affitins with DTT were purified using PD10 Sephadex column (Amersham Biosciences Europe, Orsay, France). PEGylated siRNA LNCs with $10 \mathrm{mM}$ malDSPE-PEG were mixed with purified Affitins under stirring at room temperature overnight. DSPE-PEG-Affitin LNCs were purified by CL4B sepharose column (Sigma Aldrich). The presence of LNCs was evidenced with turbidity lecture at $580 \mathrm{~nm}$ and Affitins by microBCA test (Thermo Fisher, Waltham, MA, USA) according to the manufacturer protocol.

\section{Characterization of siRNA LNCs}

Size and zeta potential. The mean diameter and polydispersity index (PDI) of LNC were measured using the dynamic light scattering (DLS) method at a backscatter angle of $173^{\circ}$. Zeta potential was measured using the laser Doppler microelectrophoresis technique with a Malvern Zetasizer® apparatus (Nano Series ZS, Malvern Instruments S.A., UK) at $25{ }^{\circ} \mathrm{C}$. The measurements were performed in triplicate, after $1: 200 \mathrm{v} / \mathrm{v}$ dilution ratio with deionized water. ${ }^{53}$

Encapsulation efficiency. A spectrophotometric method based on a work recently described by David et al., was used to evaluate the encapsulation efficiency (EE, \%). ${ }^{10}$ Briefly, siRNA LNCs were mixed with chloroform and water to separate hydrophilic and lipophilic components, respectively. Sodium hydroxide was added to destabilize lipoplexes, and finally absolute ethanol was added to destroy the LNCs. After two centrifugations, four fractions were obtained: free siRNA, free lipoplexes, encapsulated siRNA and encapsulated lipoplexes into LNCs. To determine the concentration of siRNA, the optical density of each sample was determined at $260 \mathrm{~nm}$ (UV-2600, Shimadzu, Noisiel, France) in triplicate conditions and compared to range curve to determine the ratio of the encapsulated part of siRNA in total siRNA detected in the formulation (EE, \%).

\section{Cell culture}

The SK-Mel28 human melanoma cell lines were grown in Roswell Park Memorial Institute (RPMI) 1640 medium (Lonza, Verviers, Belgium) supplemented with $10 \%$ fetal bovine serum (Lonza, Verviers, Belgium), 1\% antibiotics (10 units of penicillin, $10 \mathrm{mg}$ of streptomycin, $25 \mu \mathrm{g}$ of amphotericin B per $\mathrm{mL}$; Sigma-Aldrich, Saint louis, USA) and 1\% non-essential amino acids (Lonza). Cell lines were cultured according to ATCC protocol and maintained at $37{ }^{\circ} \mathrm{C}$ in a humidified atmosphere with $5 \% \mathrm{CO}_{2}$.

\section{Cellular uptake}

Flow cytometry. In order to discriminate the cell wall adsorbed from internalized siRNA LNCs into melanoma SKMel28 cells, a Alexa488 quenching trypan blue assay was performed. ${ }^{54,55}$

Melanoma cells were seeded onto 6-well plates at the density of $50 \times 10^{4}$ cells per well and precultured overnight. Before transfection, the medium was changed to a fresh medium, without serum. The cells were treated with double fluorescent
siRNA LNCs (DiD and Alexa488 siRNA) at siRNA concentration of $0.25 \mu \mathrm{g} \mathrm{mL}{ }^{-1}$ for $4 \mathrm{~h}$ to $48 \mathrm{~h}$. Immunostaining was performed to evaluate the internalization of LNCs and siRNA into SK-Mel28 cells. After kinetic point (4, 8, 24 and $48 \mathrm{~h}$ ), cells were washed twice with PBS (Lonza) and incubation with trypan blue (Lonza) was performed during $1 \mathrm{~min}$ following by three PBS washing. Cell suspensions were fixed with PBS/azide $0.02 \%$ /formaldehyde $1 \%$, protected from light and stored at $4{ }^{\circ} \mathrm{C}$. Analyses were performed with a FACScalibur flow cytometer (BD Bioscience, San José, USA) in collaboration with PACeM platform (Angers).

Fluorescence microscopy. Melanoma cells were seeded onto CC2 Labtek 4 chambers (Dominique Dutcher, France) at the density of $50 \times 10^{4}$ cells per chamber and precultured overnight. Before transfection, the medium was changed to a fresh medium, without serum. The cells were treated with double fluorescent siRNA LNCs (DiD and Alexa488 siRNA) at siRNA concentration of $0.25 \mu \mathrm{g} \mathrm{mL}{ }^{-1}$ for $4 \mathrm{~h}$ to $48 \mathrm{~h}$. To stop the reaction, cells were incubated with a solution of paraformaldehyde $4 \%$ at $4{ }^{\circ} \mathrm{C}$. Cells were then washed twice with PBS $1 \times$ (Lonza) and incubation with DAPI (Sigma, Saint-Louis, MO, États-Unis) was performed during 1 min following by three PBS washing. Cells were analyzed under a fluorescence microscope (Axioscope ${ }^{\circledR} 2$ optical, Zeiss, Le Pecq, Germany).

\section{Fluorescence confocal spectral imaging (FCSI)}

To follow the nanocarrier behavior in the presence of melanoma cells, siRNA-loaded LNCs were formulated with three different fluorochromes. Two fluorescent probes, DiI and DiD were used respectively as donor and acceptor partners to obtain a FRET (Fluorescence/Förster Resonance Energy Transfer) signal. DiI $\left(\lambda_{\mathrm{ex}}=549 \mathrm{~nm}, \lambda_{\mathrm{em}}=565 \mathrm{~nm}\right)$ was excited with a $488 \mathrm{~nm}$ laser. With this excitation wavelength, the DiD fluorescence $\left(\lambda_{\mathrm{ex}}=644 \mathrm{~nm}, \lambda_{\mathrm{em}}=665 \mathrm{~nm}\right)$ can mainly result from FRET. To observe FRET, the LNCs were co-loaded with DiI : DiD mixture at $2: 1$ molar ratio. In addition, the LNCs were loaded with siRNA conjugated to Alexa488 fluorophore $\left(\lambda_{\text {ex }}=488 \mathrm{~nm}\right.$, $\lambda_{\text {em }}=525 \mathrm{~nm}$ ). Therefore, with the triple-labelled LNCs, we simultaneously excited the DiI/DiD FRET couple and the green fluorescence of Alexa488 in order to follow both LNC integrity and siRNA distribution.

SK-Mel28 cells were seeded onto CC2 Labtek 4 chambers (Dominique Dutcher, France) at the density of $30 \times 10^{3}$ cells per chamber and precultured for $96 \mathrm{~h}$. The medium was then replaced by a suspension of triple-labelled siRNA LNCs in the culture medium without serum at the concentration of $0.50 \mu \mathrm{g}$ $\mathrm{mL}^{-1}$ of siRNA and the cells incubated for $0.5,3.5,5$, or $15 \mathrm{~h}$ at $37{ }^{\circ} \mathrm{C}, 5 \% \mathrm{CO}_{2}$. After treatment, SK-Mel28 cells were washed twice in HBSS supplemented with $\mathrm{Ca}^{2+}$ and $\mathrm{Mg}^{2+}$. Then the media chambers were removed, some HBSS supplemented with $\mathrm{Ca}^{2+}$ and $\mathrm{Mg}^{2+}$ was added to keep the cells alive during the fluorescence confocal spectral imaging (FCSI), and finally the cells were recovered by a lamella.

The FCSI measurements were carried out using a LabRam confocal microspectrometer (Horiba Jobin Yvon, Villeneuve d'Ascq, France) equipped with an automated $X-Y-Z$ scanning stage, a low dispersion grating (300 grooves per $\mathrm{mm}$ ) and an air- 
cooled CCD detector. The excitation wavelength used was a $488 \mathrm{~nm}$ line of an $\mathrm{Ar}^{+}$laser (Melles Griot, France) and collected in a confocal mode, through the $50 \times$ LWD objective (NA 0.5). For each cell analysis, an equatorial optical section $(x-y$ plane situated at half-thickness of the cell) was scanned with a step of $0.7 \mu \mathrm{m}$ that provided maps containing typically $\approx 900$ spectra. The cellular auto-fluorescence was completely neglected, because of the absence of any significant fluorescence of the untreated cells under the conditions used (laser power on the sample ca. $5 \mu \mathrm{W}, 0.03 \mathrm{~s}$ per spectrum). No sample photodegradation was observed.

Both acquisition ( $n=3$ to 5 ) and treatment of multispectral maps were performed with LabSpec software. Subcellular LNC and siRNA distribution maps were established via analysis of both the intensity and shape of intracellular fluorescence spectra. Briefly, each experimental spectrum was fitted using the least-squares method to a sum of three reference spectra described below. The fitting errors were below 5\% (typically 2$4 \%)$.

\section{Plasma protection}

SiRNA, siRNA LNCs and surface modified formulations containing $30 \mu \mathrm{g}$ siRNA were incubated for different time intervals in $50 \%(\mathrm{v} / \mathrm{v})$ Normal Human Serum (NHS) at $37{ }^{\circ} \mathrm{C} .{ }^{30}$ Following incubation, samples were stored at $-20{ }^{\circ} \mathrm{C}$. Electrophoresis into $1 \%(\mathrm{~m} / \mathrm{v})$ agarose gels in TBE buffer containing ethidium bromide (EtB) was performed at $125 \mathrm{~V}$ for $20 \mathrm{~min}$ and the resulting gels were photographed under UV light.

\section{In vivo experiments}

Orthotopic mouse model of melanoma. Six- to eight-weekold female, nude NMRI mice (Elevage Janvier, France) were housed and maintained at the SCAHU; they were processed in accordance with the laboratory animal care guidelines (NIH publication 85-23, revised 1985) and with the agreement of the national ethic committee (national authorization no. 01315.01, France). Tumor bearing mice were prepared by injecting subcutaneously a suspension of $3 \times 10^{6}$ SK-Mel28 melanoma cells in $100 \mu \mathrm{L}$ of RPMI medium into the right flank of athymic nude NMRI mice (6-8 week-old females, 20-24 g).

Biofluorescence imaging. Non-invasive fluorescence imaging (BFI) was performed $15 \mathrm{~min}, 30 \mathrm{~min}, 45 \mathrm{~min}, 1 \mathrm{~h}, 3 \mathrm{~h}, 5 \mathrm{~h}, 8 \mathrm{~h}$, $24 \mathrm{~h}$ and $48 \mathrm{~h}$ post-injection, using the BFI system of the Maestro $^{\mathrm{TM}}$ II (CRi, Woburn, USA) equipped with cooled CCD camera and lipid crystal tunable filter (LCTF) and driven with the Maestro 2 software (Cambridge Research \& Instrumentation, Inc, USA). Considering the fluorescence characteristics of the DiD fluorescent tag used to follow the LNCs, wavelength spectrum chosen was from 600 to $700 \mathrm{~nm}$. In parallel, the light beam was kept constant for each measurement, which was ideal with the ringlight, and epi-illumination. As the ringlight was always set at the same intensity, the excitation energy on the sample would always stay the same. Each mouse was anesthetized with a $4 \%$ air-isoflurane blend. Once placed in the acquisition chamber, the anesthesia of the mice was maintained with a $2 \%$ air-isoflurane mixture throughout the experiment, as described above. With the BFI system, the fluorescence acquisition time was of $5 \mathrm{~s}$. Animals were sacrificed at last time acquisition ( $48 \mathrm{~h}$ ) and dissection of organs was performed to individually evaluate the fluorescence. All data were analyzed with the Maestro II software to obtain the average fluorescence signal for each organ (photon per $\mathrm{s}$ per $\mathrm{cm}^{2}$ ). Ratio between auto-fluorescence of control organ (without injection of fluorescent LNCs) and fluorescence of organs receiving the different DiD LNCs were performed to compare all formulations.

\section{Statistical analysis}

Comparisons between all groups, supposed with normal distribution, were performed using a classical analysis of variance (one-way ANOVA) followed by a Tukey's post hoc analysis. The encapsulation efficiencies for modified LNCs and nonmodified LNCs were compared using a $t$-test. Statistical significance was ascribed to a threshold $p$-value of $0.05\left({ }^{*} p \leq 0.05 ; * * p\right.$ $\leq 0.01 ; * * p \leq 0.001)$.

\section{Conclusions}

Lipid nanocapsules (LNCs) previously demonstrated their capacity to efficiently encapsulate siRNAs. In this study, FRET experiments proved the efficient time-dependency delivery of siRNA into the cytosol of human melanoma cells, thanks to a strong interaction/fusion of LNCs with the cell membranes. In addition, different surface modifications were performed. While the tested DSPE-PEG LNCs were not able to penetrate into melanoma cells, results showed the efficient internalization of siRNA loaded LNCs modified by TE-PEG. The biodistribution on melanoma mice model showed the best tumor targeting for pegylated LNCs at $48 \mathrm{~h}$, notably for TE-PEG LNCs. Moreover, this TE-PEG, recently modified thanks to the addition of $\mathrm{pH}$ sensitive link between the PEG and TE molecule, could represent an innovative moiety to develop smart nanomedicines. Finally, the results obtained with an Affitin are encouraging, as its presence on LNCs did not induce in vivo undesirable side effect. The use of a melanoma specific Affitin combined with an optimized balance of DSPE or TE-PEG/Affitin would allow an efficient smart targeting.

\section{Conflicts of interest}

There are no conflicts to declare.

\section{Abbreviations}
BFI
DiD
Biofluorescence imaging
1,1'-Dioctadecyl-3,3,3', $3^{\prime}$ -
tetramethylindodicarbocyanine perchlorate
DiI 1, $\quad 1$ '-Dioctadecyl-3,3,3', $3^{\prime}$ - tetramethylindocarbocyanine perchlorate
DOPE 1,2-Dioleoyl-sn-glycero-3-phosphoethanolamine
DOTAP 1,2-Dioleoyl-3-trimethylammoniumpropane 
DSPE-PEG 1,2-Distearoyl-sn-glycero-3-

phosphoethanolamine- $N$ -

[methoxy(polyethyleneglycol)-2000]

DTT Dithiothreitol

FRET Fluorescence/Förster resonance energy transfer

i.v. Intravenous

LNCs Lipid nanocapsules

malDSPE- 1,2-Distearoyl-sn-glycero-3-

PEG phosphoethanolamine- $N$ -

[maleimide(polyethyleneglycol)-2000]

SiRNA Small interfering RNA

TE-PEG Tetraether-polyethylene glycol

\section{Acknowledgements}

The authors would like to thank the SFR ICAT and the PACEM platform for flow cytometry and especially Catherine Guillet for her expertise in this field. We are also grateful to Dr Joel Eyer and Dr Franck Letournel for their collaboration in using the Maestro II, Dr Anne Clavreul for her expertise in fluorescence microscopy, and Dr Guillaume Bastiat for his help on statistical analysis. This work was supported by special grants from the "Association de la Recherche contre le Cancer” (Reference no.: SFI20121205972) and by "La Ligue contre le cancer 49 et 35" (Reference no.: R12164NN). P. Resnier and C. Passirani are the inventors of a French patent (FR 4185991, 24 Sept 2014) about the formulation of stable siRNA LNCs, owned by University of Angers. F. Pecorari is inventor of a patent application (PCT/IB2007/004388), owned by Institut Pasteur and Centre National de Recherche Scientifique (CNRS), related to generation of Affitins.

\section{References}

1 A. Fire, et al., Potent and specific genetic interference by double-stranded RNA in Caenorhabditis elegans, Nature, 1998, 391, 806-811.

2 Q. Lin, J. Chen, Z. Zhang and G. Zheng, Lipid-based nanoparticles in the systemic delivery of siRNA, Nanomedicine, 2014, 9, 105-120.

3 G. Tiram, A. Scomparin, P. Ofek and R. Satchi-Fainaro, Interfering cancer with polymeric siRNA nanomedicines, $J$. Biomed. Nanotechnol., 2014, 10, 50-66.

4 Z. Du, M. M. Munye, A. D. Tagalakis, M. D. I. Manunta and S. L. Hart, The Role of the Helper Lipid on the DNA Transfection Efficiency of Lipopolyplex Formulations, Sci. Rep., 2014, 4, 7107.

5 D. C. Litzinger and L. Huang, Phosphatidylethanolamine liposomes: drug delivery, gene transfer and immunodiagnostic applications, Biochim. Biophys. Acta, 1992, 1113, 201-227.

6 P. Resnier, T. Montier, V. Mathieu, J.-P. Benoit and C. Passirani, A review of the current status of siRNA nanomedicines in the treatment of cancer, Biomaterials, 2013, 34, 6429-6443.
7 S. David, B. Pitard, J.-P. Benoît and C. Passirani, Non-viral nanosystems for systemic siRNA delivery, Pharmacol. Res., 2010, 62, 100-114.

8 M. Morille, C. Passirani, A. Vonarbourg, A. Clavreul and J.-P. Benoit, Progress in developing cationic vectors for non-viral systemic gene therapy against cancer, Biomaterials, 2008, 29, 3477-3496.

9 P. Resnier, et al., Efficient in vitro gene therapy with PEG siRNA lipid nanocapsules for passive targeting strategy in melanoma, Biotechnol. J., 2014, 9, 1389-1401.

10 S. David, et al., siRNA LNCs-a novel platform of lipid nanocapsules for systemic siRNA administration, Eur. J. Pharm. Biopharm., 2012, 81, 448-452.

11 P. Resnier, et al., EGFR siRNA lipid nanocapsules efficiently transfect glioma cells in vitro, Int. J. Pharm., 2013, 454, 748755.

12 M. Morille, et al., Long-circulating DNA lipid nanocapsules as new vector for passive tumor targeting, Biomaterials, 2010, 31, 321-329.

13 S. David, et al., In vivo imaging of DNA lipid nanocapsules after systemic administration in a melanoma mouse model, Int. J. Pharm., 2012, 423, 108-115.

14 H. Maeda, J. Wu, T. Sawa, Y. Matsumura and K. Hori, Tumor vascular permeability and the EPR effect in macromolecular therapeutics: a review, J. Control. Release, 2000, 65, 271-284.

15 C. Laine, et al., Folate-equipped pegylated archaeal lipid derivatives: synthesis and transfection properties, Chemistry, 2008, 14, 8330-8340.

16 T. Benvegnu, L. Lemiegre and S. Cammas-Marion, New generation of liposomes called archaeosomes based on natural or synthetic archaeal lipids as innovative formulations for drug delivery, Recent Pat. Drug Deliv. Formulation, 2009, 3, 206-220.

17 J. Barbeau, S. Cammas-Marion, P. Auvray and T. Benvegnu, Preparation and Characterization of Stealth Archaeosomes Based on a Synthetic PEGylated Archaeal Tetraether Lipid, J. Drug Delivery, 2011, 2011, 396068.

18 A. Jhaveri, P. Deshpande and V. Torchilin, Stimuli-sensitive nanopreparations for combination cancer therapy, $J$. Control. Release, 2014, 190, 352-370.

19 V. Kalichuk, et al., The archaeal "7 kDa DNA-binding" proteins: extended characterization of an old gifted family, Sci. Rep., 2016, 6, 37274.

20 G. Behar, et al., Tolerance of the archaeal Sac7d scaffold protein to alternative library designs: characterization of anti-immunoglobulin G Affitins, Protein Eng. Des. Sel., 2013, 26, 267-275.

21 A. Correa, et al., Potent and specific inhibition of glycosidases by small artificial binding proteins (affitins), PLoS One, 2014, 9, e97438.

22 B. Mouratou, et al., Remodeling a DNA-binding protein as a specific in vivo inhibitor of bacterial secretin PulD, Proc. Natl. Acad. Sci. U. S. A., 2007, 104, 17983-17988.

23 G. Behar, S. Pacheco, M. Maillasson, B. Mouratou and F. Pecorari, Switching an anti-IgG binding site between archaeal extremophilic proteins results in Affitins with 
enhanced pH stability, J. Biotechnol., 2014, 192(Pt A), 123129.

24 G. Behar, A. Renodon-Corniere, B. Mouratou and F. Pecorari, Affitins as robust tailored reagents for affinity chromatography purification of antibodies and nonimmunoglobulin proteins, J. Chromatogr. A, 2016, 1441, $44-51$.

25 C. S. M. Fernandes, et al., Affitins for protein purification by affinity magnetic fishing, J. Chromatogr. A, 2016, 1457, 50-58.

26 V. Kalichuk, et al., A novel, smaller scaffold for Affitins: Showcase with binders specific for EpCAM, Biotechnol. Bioeng., 2018, 115, 290-299.

27 S. Pacheco, G. Behar, M. Maillasson, B. Mouratou and F. Pecorari, Affinity transfer to the archaeal extremophilic Sac7d protein by insertion of a CDR, Protein Eng. Des. Sel., 2014, 27, 431-438.

28 G. Behar, et al., Whole-bacterium ribosome display selection for isolation of highly specific anti-Staphyloccocus aureus Affitins for detection- and capture-based biomedical applications, Biotechnol. Bioeng., 2019, 116, 1844-1855.

29 G. Grazia, I. Penna, V. Perotti, A. Anichini and E. Tassi, Towards combinatorial targeted therapy in melanoma: from pre-clinical evidence to clinical application (review), Int. J. Oncol., 2014, 45, 929-949.

30 W. J. Kim, et al., Anti-angiogenic inhibition of tumor growth by systemic delivery of, J. Control. Release, 2006, 114, 381388.

31 E. Bourseau-Guilmain, et al., Development and characterization of immuno-nanocarriers targeting the cancer stem cell marker AC133, Int. J. Pharm., 2012, 423, 93-101.

32 B. Heurtault, P. Saulnier, B. Pech, J.-E. Proust and J.-P. Benoit, A novel phase inversion-based process for the preparation of lipid nanocarriers, Pharm. Res., 2002, 19, 875-880.

$33 \mathrm{~J}$. Hureaux, et al., Lipid nanocapsules: ready-to-use nanovectors for the aerosol delivery of paclitaxel, Eur. J. Pharm. Biopharm., 2009, 73, 239-246.

34 E. Roger, F. Lagarce, E. Garcion and J.-P. Benoit, Reciprocal competition between lipid nanocapsules and P-gp for paclitaxel transport across Caco-2 cells, Eur. J. Pharm. Sci., 2010, 40, 422-429.

35 A. Vonarbourg, et al., The encapsulation of DNA molecules within biomimetic lipid nanocapsules, Biomaterials, 2009, 30, 3197-3204.

36 Y. Xie, et al., PEGylated carboxymethyl chitosan/calcium phosphate hybrid anionic nanoparticles mediated hTERT siRNA delivery for anticancer therapy, Biomaterials, 2014, 35, 7978-7991.

37 A. Paillard, F. Hindre, C. Vignes-Colombeix, J.-P. Benoit and E. Garcion, The importance of endo-lysosomal escape with lipid nanocapsules for drug subcellular bioavailability, Biomaterials, 2010, 31, 7542-7554.

38 A. Elouahabi and J.-M. Ruysschaert, Formation and intracellular trafficking of lipoplexes and polyplexes, Mol. Ther., 2005, 11, 336-347.
39 Y. Ma, et al., Enhanced bactericidal potency of nanoliposomes by modification of the fusion activity between liposomes and bacterium, Int. J. Nanomed., 2013, 8, 2351-2360.

40 A.-L. Laine, et al., Conventional versus stealth lipid nanoparticles: formulation and in vivo fate prediction through FRET monitoring, J. Control. Release, 2014, 188, 1-8.

41 C. Rangger, et al., Influence of PEGylation and RGD loading on the targeting properties of radiolabeled liposomal nanoparticles, Int. J. Nanomed., 2012, 7, 5889-5900.

42 Y. Li, M. Kröger and W. K. Liu, Endocytosis of PEGylated nanoparticles accompanied by structural and free energy changes of the grafted polyethylene glycol, Biomaterials, 2014, 35, 8467-8478.

43 E. C. Cho, Q. Zhang and Y. Xia, The effect of sedimentation and diffusion on cellular uptake of gold nanoparticles, Nat. Nanotechnol., 2011, 6, 385-391.

44 W. Dai, et al., A comprehensive study of iRGD-modified liposomes with improved chemotherapeutic efficacy on B16 melanoma, Drug Deliv., 2015, 22, 10-20.

45 A. D. Friedman, S. E. Claypool and R. Liu, The smart targeting of nanoparticles, Curr. Pharm. Des., 2013, 19, 6315-6329.

46 N. T. Huynh, E. Roger, N. Lautram, J.-P. Benoit and C. Passirani, The rise and rise of stealth nanocarriers for cancer therapy: passive versus active targeting, Nanomedicine, 2010, 5, 1415-1433.

47 Y. Kato, et al., Acidic extracellular microenvironment and cancer, Cancer Cell Int., 2013, 13, 89.

$48 \mathrm{~K}$. Möller, et al., Highly efficient siRNA delivery from coreshell mesoporous silica nanoparticles with multifunctional polymer caps, Nanoscale, 2016, 7, 4007.

49 S. Guo, et al., Enhanced Gene Delivery and siRNA Silencing by Gold Nanoparticles Coated with Charge-Reversal Polyelectrolyte, ACS Nano, 2010, 4, 5505-5511.

50 L. Kirkpatrick, et al., Carbon Nanotubes: Solution for the Therapeutic Delivery of siRNA?, Materials, 2012, 5, 278-301.

51 G. Cavallaro, C. Sardo, E. F. Craparo, B. Porsio and G. Giammona, Polymeric nanoparticles for siRNA delivery: production and applications, Int. J. Pharm., 2017, 525, 313333.

52 T. S. Zatsepin, Y. V. Kotelevtsev and V. Koteliansky, Lipid nanoparticles for targeted siRNA delivery - going from bench to bedside, Int. J. Pharm., 2016, 11, 3077-3086.

53 G. Bastiat, et al., A new tool to ensure the fluorescent dye labeling stability of nanocarriers: a real challenge for fluorescence imaging, J. Control. Release, 2013, 170, 334-342.

54 E. van Bracht, et al., Enhanced cellular uptake of albuminbased lyophilisomes when functionalized with cellpenetrating peptide TAT in HeLa cells, PloS one, 2014, 9, e110813.

55 G. K. Srivastava, et al., Trypan Blue staining method for quenching the autofluorescence of RPE cells for improving protein expression analysis, Exp. Eye Res., 2011, 93, 956-962. 